\title{
Dip coating in the presence of a substrate-liquid interaction potential
}

\author{
R. Krechetnikov ${ }^{a)}$ and G. M. Homsy \\ Department of Mechanical Engineering, University of California, Santa Barbara, California 93106
}

(Received 24 March 2005; accepted 14 September 2005; published online 19 October 2005)

\begin{abstract}
In this work we investigate theoretically the Landau-Levich problem of dip coating in the presence of a strong interaction potential normal to the substrate. This study is motivated by dip coating at very low capillary numbers when the deposited film thickness is less than $1 \mu \mathrm{m}$ and such interaction forces become important. The objective of this work is to demonstrate that in the presence of an extra body force the solution procedure differs significantly from the classical one and leads to substantial deviations from the Landau-Levich law for the entrained film thickness. In particular, attractive potentials produce film thickening and the resulting film thickness is independent of speed to lowest order. Repulsive potentials bring about more complicated behavior and lead either to films whose thickness is also independent of speed, or to a modification of the leading order constant in the classical $\mathrm{Ca}^{2 / 3}$ law. Demonstration of these effects is given for a model potential. The analysis is generally applicable to many physical situations when there is an interaction between a coating liquid and a substrate, e.g., dip coating of ferromagnetic liquids on magnetic substrates, or dip coating of liquids carrying charges. (c) 2005 American Institute of Physics.
\end{abstract}

[DOI: $10.1063 / 1.2107927$ ]

\section{INTRODUCTION}

The simplest coating process-film deposition by withdrawing a substrate from a liquid bath-remains one of the most fundamentally important coating processes. The basic question of the dependence of film thickness upon the withdrawal speed $U$, the gravitational acceleration $g$, and the physical properties of the fluid, i.e., fluid density $\rho$, viscosity $\mu$, and surface tension $\sigma$, was first answered by Landau and Levich $^{1}$ for dip coating from an infinite bath in the low capillary number limit (when surface forces dominate viscous ones). Their analysis, now recognized as a matched asymptotic expansion combined with a lubrication approximation, hinges on the geometrical matching of the curvature of the static meniscus to the zero curvature in the thin film region through a transition region, and yields the well-known expression for the film thickness

$$
\bar{h}_{\infty}=0.945 l_{c} \mathrm{Ca}^{2 / 3}, \quad \mathrm{Ca}=\frac{\mu U}{\sigma} .
$$

Here the relevant length scale is the capillary length $l_{c}$ $=\sqrt{\sigma_{l g} / \rho g}$, where we use standard convention for the indexes: $l$ for liquid, $g$ for gas, and $s$ for solid. It is known that this law is distorted when the static meniscus is modified. This can be achieved for example by modifying the geometry of the unbounded flat substrate to a finite width strip ${ }^{2}$ or to a finite radius rod. ${ }^{3}$ One can expect that the shape of the static meniscus can also be modified if there is an interaction between the substrate and coating liquid.

The latter case, i.e., when there is a body force perpendicular to the gravity field, has not been systematically studied in the literature. The only relevant work is by Wilson ${ }^{4}$

\footnotetext{
${ }^{a)}$ Current address: Caltech, Division of Engineering and Applied Science, Pasadena, CA 91125.
}

who addressed asymptotically the case of a plate inclined at an angle $\alpha$ with respect to gravity such that the angle between the liquid level in the bath and the film is obtuse ( $\alpha$ $>0$ ), thus yielding the expression

$$
\bar{h}_{\infty}=\frac{0.945}{\sqrt{1-\sin \alpha}} l_{c} \mathrm{Ca}^{2 / 3}+O(\mathrm{Ca}),
$$

which diverges at $\alpha=\pi / 2$. In this problem there are components of the body force both parallel and perpendicular to the substrate, and the condition $\alpha>0$ is critical for matching the thin film region with the static meniscus. We refer to the case $\alpha>0$ as an attractive interaction since the component of gravity normal to the substrate, $g \sin \alpha$, is positive. However as we will see, the case treated by Wilson ${ }^{4}$ is fundamentally different from the case of a substrate withdrawn vertically (with respect to gravity) in the presence of an arbitrary potential of interaction between the substrate and liquid. In particular, we will demonstrate that in the case of repulsive interaction (which in the context of Wilson's problem would correspond to $\alpha<0$ ) the above-mentioned matching is not possible for some range of parameters. We show that the classical matching procedure is also invalidated in the attractive interaction case in view of the intrinsic physical difference between this problem and the standard Landau-Levich problem, discussed in Secs. III and IV.

The context for our work is the case of dip coating at very low capillary numbers, when the entrained film thickness is less than $1 \mu \mathrm{m}$ (the question of the existence and stability of the film is not addressed here). At these scales the interaction of the liquid and substrate through London-van der Waals forces, diffuse double layers, and structural forces comes into play. For the silica/water system, for example, it is known that electrical double-layer forces dominate over the London-van der Waals forces for film thicknesses greater 
$300 \AA$ A. This fact was verified experimentally ${ }^{5,6}$ by film thickness measurements in the presence of salt(s), which is known to suppress the double-layer interaction-the film thickness decreases with an increase of salt concentration and of valency of the cation. The nature of the double layer at the silica/water interface is due to dissociation of terminal silanol groups, the degree of which depends on the water type (in view of the aggressive nature of deionized water, the dissociation is the strongest): silica acquires a negative charge of $0.32 \mathrm{mC} / \mathrm{m}^{2}$ with double layer thickness $\bar{l}_{D}$ $=0.275 \mu \mathrm{m} .{ }^{7,8}$ Rough or porous silica surfaces lead to much higher charge. ${ }^{9}$ As noted, the range of the London-van der Waals forces is typically $\sim 0.03 \mu \mathrm{m}$, whereas the interaction distance of double layers is the Debye length, $\bar{l}_{D} \sim 0.3 \mu \mathrm{m}$. However, their strong effect on the film stability was found experimentally at very great thicknesses, $10^{-2} \mathrm{~cm}$, in the experiments of Padday ${ }^{10}$ on film rupture and theoretically confirmed by Mitlin, ${ }^{11}$ who described the de-wetting theory in analogy with the Cahn theory of spinodal decomposition. The effect of the inclusion of aqueous salt and surfactants ${ }^{10}$ suggests that the electric double layer is the origin of the long range forces. In addition, a number of experiments indicate a strong dependence of the film thickness on the presence of a charge on a substrate: introduction of electrostatic charge on a clean glass rod by rubbing with rabbit fur ${ }^{12}$ led to substantial deviations from theoretical predictions of Landau-Levich: the higher the charge the lower the thickness. Therefore, it is possible that deviations from the Landau-Levich solution (1) due to electrical forces of microscopic origin can be observed for thicker films.

Motivated by some of the above general considerations Teletzke et al. ${ }^{13}$ focused on the so-called transition region and assumed that the solution there obeyed a Landau-Levich equation augmented with an extra body force originating from London-van der Waals interactions. Their aim was to generalize Bretherton's analysis ${ }^{14}$ of the closely related problem of film deposition due to meniscus propagation in a capillary tube and to explain discrepancies between Bretherton's theory and experiments at low capillary numbers when the films are thin. As we demonstrate in Sec. II, the effect of such terms on the Landau-Levich solution appears only in the next order in $\mathrm{Ca}$, whereas in the strong potential limit the static meniscus affects the solution at leading order. Besides the relevance of our analysis to dip coating of very thin films, the Landau-Levich model with an extra body force is of general physical interest in other physical situations including coating with ferromagnetic liquids, or liquids carrying net charges.

\section{PROBLEM FORMULATION}

In order to make the exposition of the theory and its difference from previous analyses clear, it is instructive to recall the roots of the Landau-Levich solution and the basic solution properties of the Young-Laplace equation. The wellknown Young equation,

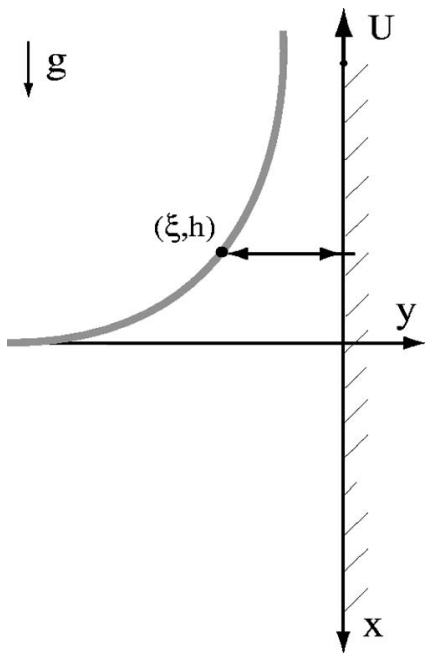

FIG. 1. Coordinate system: $h \leqslant 0$ and $\xi \leqslant 0$.

$$
\sigma_{l g} \cos \theta_{0}=\sigma_{s g}-\sigma_{s l},
$$

provides an explanation and a boundary condition for capillary rise of a static meniscus - the contact point rises, deforming the interface to equilibrate the tensions of three interfaces according to (3). In the neighborhood of the contact point the mass of liquid is negligible (for negligibly thin film adherence force dominates gravity), so that the contact angle defined by (3) is unaffected by gravity. The interface assumes a shape necessary to equilibrate the hydrostatic and capillary pressures and obeys the Young-Laplace equation

$$
p-\sigma_{l g} \kappa=0, \kappa=\frac{h_{\xi \xi}}{\left(1+h_{\xi}^{2}\right)^{3 / 2}},
$$

subject to the boundary conditions of a flat interface away from substrate and a prescribed contact angle where the meniscus intersects the plate.

We define bulk coordinates by $(x, y)$, and interface coordinates by $(\xi, h)$ with $\xi=x$ being a parameterization as seen in Fig. 1. With this definition of the interface (cf. Fig. 1), $h$ $<0, h_{\xi}<0, h_{\xi \xi}<0$. Now consider the entrainment of a film due to motion of the solid boundary. We work with nondimensional variables, defined by $\mathbf{x} \rightarrow l_{c} \mathbf{x}, \quad \mathbf{v} \rightarrow U \mathbf{v}, \quad p$ $\rightarrow \sqrt{\rho g \sigma_{l g}} p$. The bath interface corresponds to $\xi \equiv x=0$. Introducing a generic potential force $\phi(x, y)$ (also scaled by $\sqrt{\rho g \sigma_{l g}}$ ), the Stokes equations are

$$
\begin{aligned}
& 0=u_{x}+v_{y}, \\
& 0=-p_{x}+1+\phi_{x}+\mathrm{Ca} \Delta u, \\
& 0=-p_{y}+\phi_{y}+\mathrm{Ca} \Delta v,
\end{aligned}
$$

augmented by the dynamic boundary conditions at the interface:

$$
p-\kappa=\frac{2 \mathrm{Ca}}{1+h_{x}^{2}}\left\{-h_{x}\left(u_{y}+v_{x}\right)+v_{y}+h_{x}^{2} u_{x}\right\},
$$




$$
0=\frac{\mathrm{Ca}}{1+h_{x}^{2}}\left\{2 h_{x}\left(v_{y}-u_{x}\right)+\left(1-h_{x}^{2}\right)\left(u_{y}+v_{x}\right)\right\},
$$

and no-slip at the wall. Although in many applications $\phi$ is small, we are interested in the strong potential limit, and therefore treat $\phi$ as an $O(1)$ quantity.

We approach the solution of this system in the low capillary regime by asymptotic matching, as classically done by Landau and Levich. ${ }^{1}$ The problem is thus decomposed into three regions: an entrained film of constant thickness, a static meniscus, and a dynamic meniscus (or transition) region, by means of which the previous two regions are matched.

As is easy to deduce, one needs to include viscous stress effects in the dynamic meniscus region (in view of the moving boundary) in the usual way. The dominant momentum balance reads

$$
0=-p_{x}+1+\phi_{x}+\mathrm{Ca} u_{y y},
$$

which, after taking into account that $p \sim h_{x x}$ as the relevant simplification of (6a) and introducing the stream function $\psi_{y}=u$, becomes

$$
0=h_{x x x}-1+\phi_{x}+\mathrm{Ca} \psi_{y y y},
$$

with associated boundary conditions

$$
\begin{aligned}
& y=0: \psi_{y}=-1, \psi=0, \\
& y=h: \psi_{y y}=0 .
\end{aligned}
$$

Assuming a weak dependence of $\phi_{x}$ on the $y$ coordinate, the resulting solution for the stream function

$$
\psi=\frac{h_{x x x}-1-\phi_{x}}{\mathrm{Ca}} \frac{y^{2}}{6}(y-3 h)-y,
$$

is evaluated at $y=h$ and equated to the value of this stream function at the interface of the constant film as $x \rightarrow-\infty$. This produces the standard thin film equation:

$$
h_{\xi \xi \xi} h^{3}+\underline{\left(1+\phi_{\xi}\right)\left(h_{\infty}^{3}-h^{3}\right)}+3 \mathrm{Ca}\left(h-h_{\infty}\right)=0,
$$

where the first term is due to capillary forces, the second underlined term results from body forces, and the third term comes from viscous stresses. When $\xi \rightarrow \infty$, the solution approaches one of constant curvature, so that $h \sim \xi^{2}$. Then for $\xi \rightarrow \infty$ we have either $h_{\xi \xi \xi \xi} \sim h^{-2}$ or, if the underlined terms are present, $h_{\xi \xi \xi \xi} \sim O(1)$. The latter is impossible, since this contradicts $h \sim \xi^{2}$. Now, making the standard transformation $\xi$ $\rightarrow \beta \tilde{\xi}, h \rightarrow h_{\infty} \tilde{h}$, we obtain

$$
\tilde{h} \widetilde{\xi \xi \xi} \tilde{h}^{3}-\underline{\left(1+\phi_{x}\right) \beta^{3} h_{\infty}^{-1}\left(\tilde{h}^{3}-1\right)}+3 \mathrm{Ca} \beta^{3} h_{\infty}^{-3}(\tilde{h}-1)=0 .
$$

In order to have a nondegenerate reduced equation with the correct asymptotic behavior, we require $\mathrm{Ca} \beta^{3} / h_{\infty}^{3}=1$, and, using the fact that $h_{\xi \xi} \sim O(1)$, we find $\beta=\mathrm{Ca}^{1 / 3}, h_{\infty}=\mathrm{Ca}^{2 / 3}$. As a result, with an accuracy $O\left(\mathrm{Ca}^{1 / 3}\right)$ neither body force contributes to the solution in the dynamic meniscus region. This is contrary to the hypothesis of Teletzke et al.,$^{13}$ and allows us to conclude that the leading order effect of the additional potential comes from the solution of the static meniscus in the region of parameter space $(\phi \sim O(1), \mathrm{Ca} \ll 1)$ under con- sideration here. We also note that the assumed weak dependence of $\phi_{x}$ on $y$ is not crucial for drawing the above conclusion. It remains valid as long as $\phi_{x} \sim O(1)$ with the only difference that in (9) there should be an integral of $\phi_{x}$ with respect to $y$. For future reference, numerical integration of the Landau-Levich equation, i.e., the previous equation with the underlined terms set to zero, with boundary conditions

$$
\widetilde{\xi} \rightarrow-\infty: \tilde{h}=1, \quad \tilde{h}_{\tilde{\xi}}=0, \quad \tilde{h}_{\tilde{\xi} \tilde{\xi}}=0
$$

yields

$$
\lim _{\tilde{\xi} \rightarrow+\infty} \tilde{h_{\tilde{\xi} \tilde{\xi}}}=1.34 .
$$

\section{SOLUTION PROCEDURE WITH AN EXTRA BODY FORCE}

Here we are interested in demonstrating the basic effect of an extra body force, without loss of generality and influence on our conclusions, we use a potential of the form ${ }^{15}$

$$
\phi(h, y)=\lambda_{0} h \exp \left(y / l_{D}\right), h, y<0,
$$

with $l_{D}$ standing for the analog of the Debye length nondimensionalized by $l_{c}$, and the sign of the nondimensional force per unit mass $\lambda_{0}$ defines the type of interaction, i.e., $\lambda_{0}<0$-attraction and $\lambda_{0}>0$-repulsion. As mentioned above, $\lambda_{0}$ and $l_{D}$ are treated here as independent $O(1)$ parameters. This choice of the potential of interaction allows the existence of a flat bath interface in view of its exponential decay away from the substrate, $y \rightarrow \infty: \phi(h, y) \rightarrow 0$, and the absence of interaction if the film thickness is zero, $h \rightarrow 0$ : $\phi(h, y) \rightarrow 0$. With this form of $\phi(h, y)$, the shape of the static meniscus interface is found from the solution of

$$
\xi-\left.\phi(h, y)\right|_{y=h}+C=\frac{h_{\xi \xi}}{\left(1+h_{\xi}^{2}\right)^{3 / 2}},
$$

which results from the nondimensional version of Eq. (4). From the consideration of the flat interface region away from the substrate it follows that the integration constant $C=0$. Integrating (14) from 0 to some arbitrary point $\xi<0$,

$$
\frac{\xi^{2}}{2}-\int_{0}^{\xi} \phi[h(\xi)] \mathrm{d} \xi=\frac{h_{\xi}}{\left(1+h_{\xi}^{2}\right)^{1 / 2}}+1,
$$

so that the point at which $h_{\xi}=0$, i.e., where the static meniscus is capable of matching with a flat film, is defined by

$$
\frac{\xi^{* 2}}{2}-\int_{0}^{\xi^{*}} \phi[h(\xi)] \mathrm{d} \xi=1 .
$$

It is notable that the point of zero tangency, $h_{\xi}=0$, exists only if the interaction potential is such that $\int_{0}^{\xi^{\xi}} \phi[h(\xi)] \mathrm{d} \xi>-1$. In the absence of $\phi$ one gets $\xi^{*}=-\sqrt{2}$, and by $(14), \quad h_{\xi \xi} \xi^{*}$ $=-\sqrt{2}$, and matching curvatures of the static and dynamic menisci where the slope vanishes $h_{\xi}=0$ produces,

$$
\bar{h}=h^{*} l_{c} \mathrm{Ca}^{2 / 3}=-\frac{1.34}{\sqrt{2}} l_{c} \mathrm{Ca}^{2 / 3},
$$

where the leading numerical coefficient, $h^{*}=-0.945$, is just a ratio of the (scaled) curvature of dynamic meniscus to that of 
the static one. Thus, in the absence of any extra body force, the matching is completed without finding the form of $h(\xi)$, even though the classical case admits complete integration in quadratures,

$$
h(\xi)=\ln (-\xi)-\ln \left[2+\sqrt{4-\xi^{2}}\right]+\sqrt{4-\xi^{2}}+\text { const. }
$$

The appearance of an arbitrary constant in (18) illustrates another important feature- the invariance of the solution $h \rightarrow h+$ const in the classical case-which is absent in the presence of a nonzero potential $\phi$. This fact has some importance consequences vis-à-vis the matching procedure. It is useful to briefly recall how the matching proceeds in the classical case from the perspective of matched asymptotic expansions. ${ }^{1}$ The outer solutions of a flat film and a static meniscus respectively are to be matched asymptotically through the dynamic meniscus or transition layer. Although conceptually straightforward, the matching is tied to the invariance of the static meniscus to an arbitrary additive constant and to the lack of a specific origin for the transition region. In the closely related Bretherton problem, application of the matching principle determines the analogous quantities and shows that the film thickness is determined by matching curvatures. ${ }^{16}$ Since the resulting film thickness is asymptotically thin, $O\left(\mathrm{Ca}^{2 / 3}\right)$, this determines the additive constant in the static meniscus solution of the present problem in such a way that the outer solution (the apparent distance between the interface and the substrate) vanishes at the point of tangency with the wall. In view of zero tangency of this point, the static meniscus appears to meet the substrate with zero apparent contact angle. See Park and Homsy ${ }^{16}$ for further discussion.

These considerations change in a fundamental way for strong interaction potentials. As already alluded, the outer static meniscus is not invariant to an arbitrary shift in $h$ and as a result, there is no guarantee that problem (14) has a solution for one endpoint fixed at $h=0$. Instead, the numerical integration of (14) demonstrates that the film thickness is determined by either (1) its value when $h_{\xi}=0$, since this is the only way to match with a flat film if the static interface does not intersect the substrate, or (2) by matching the curvatures of the dynamic and static menisci, when the static interface does approach the point $h=0$ but does not exhibit a tangency point $h_{\xi}=0$. Thus, in the first case the film thickness is independent of speed at leading order. The matching principle then presumably fixes the origin for the transition layer, as well as providing $\mathrm{Ca}$ dependence corrections to the film thickness, but we do not pursue these details here. In the second case when the static meniscus meets the plate at a finite angle, the film thickness is $\mathrm{Ca}$ dependent at leading order. In this case, matching follows a more classical route with the curvature determining the film thickness, which again becomes dependent on the withdrawal speed.

In view of the absence of analytical solution for (14), pursuing these issues in detail requires numerical integration. Before doing so, it is instructive to understand the nature of the classical problem (14) with $\phi \equiv 0$. This is a nonlinear free boundary problem with conditions at the ends (we redesignate $\xi \rightarrow-\xi, h \rightarrow-h$ for convenience):

$$
\xi \rightarrow 0: h \rightarrow-\ln \xi ; \xi \rightarrow \xi^{*}: h_{\xi} \rightarrow 0,
$$

where $\xi^{*}$ is not known a priori. The particular nonlinear character of the problem and of the boundary conditions allows for a unique solution of this underdetermined formulation. From a numerical standpoint, it is more constructive to consider this as an initial value problem with an initial condition, that is asymptotically correct,

$$
\xi \rightarrow 0: h \rightarrow-\ln \xi+D,
$$

where $D$ is an arbitrary constant. The integration is then performed up to the point $\xi^{*}$, where $h_{\xi}=0$. The above-mentioned asymptotic behavior (20) is applicable to the case when an interaction potential is present and can be obtained independently of the analytic expression (18) and directly from (14) by the observation that $\left|h_{\xi}\right|,\left|h_{\xi \xi}\right| \gg 1$ and understanding that the bath interface should approach the flat form. Therefore, the only asymptotic behavior is logarithmic, as $\xi \rightarrow-0$.

To avoid the singularity in (20) and achieve numerical accuracy it is convenient to transform the solution into a new function which has bounded behavior, $h(\xi)=\eta(\xi) \ln \xi$, so that initial conditions for $\eta(\xi)$ becomes

$$
\eta(\xi)=-1+\frac{D}{\ln \xi}, \eta^{\prime}=0 .
$$

This, however, permits integration only up to some point $\xi$ $<1$, since at $\xi=1$ one encounters a singularity of $\eta(\xi)$. Thus we numerically integrated the equation for $\eta(\xi)$ with initial conditions (21) on the interval $\xi \in[0,0.5]$ :

$$
\begin{aligned}
& \xi^{2} \ln \xi \eta^{\prime \prime}=\eta-2 \xi \eta^{\prime}+\left(1-\frac{\phi}{\xi}\right)\left[\xi^{2}+\left(\eta+\xi \ln \xi \eta^{\prime}\right)^{2}\right]^{3 / 2}, \\
& \xi \rightarrow 0: \eta \rightarrow-1, \eta^{\prime} \rightarrow 0,
\end{aligned}
$$

and continued up to $\xi^{*}$ in the original variables (14). The unknown constant $D$ is found by requiring that (16) is satisfied exactly. Thus the solution of the problem amounts to a shooting procedure: we integrate (22) and (14) up to the point of tangency, $h_{\xi}=0$, and adjust $D$ to satisfy (16). The integration is performed with sixth-order Runge-Kutta and a tolerance of $10^{-10}$, and validation of (16) is sought. The study of convergence is performed by refining the step size and tolerance. The algorithm has been tested on the classical case by verifying $\xi^{*}=h_{\xi \xi}=\sqrt{2}$, which we obtain to machine accuracy. The impossibility of solving (14) with one of the endpoints to be at the substrate, $h^{*}=0$, has been demonstrated numerically using the discussed algorithm. ${ }^{17}$

\section{RESULTS}

Attractive interaction, $\lambda_{0}<0$. Figure 2 demonstrates the change in the profile shape for fixed $\lambda_{0}$ with increase of the characteristic nondimensional length $l_{D}$. The $O(1)$ values of $l_{D}$ are relevant to coating of ferromagnetic liquids for example, whereas small values of $l_{D}$ are applicable to dip coating of regular liquids with relatively thick double layers. As a result of the lack of invariance, the film takes a specific thickness at the point where $h_{\xi}=0$. 


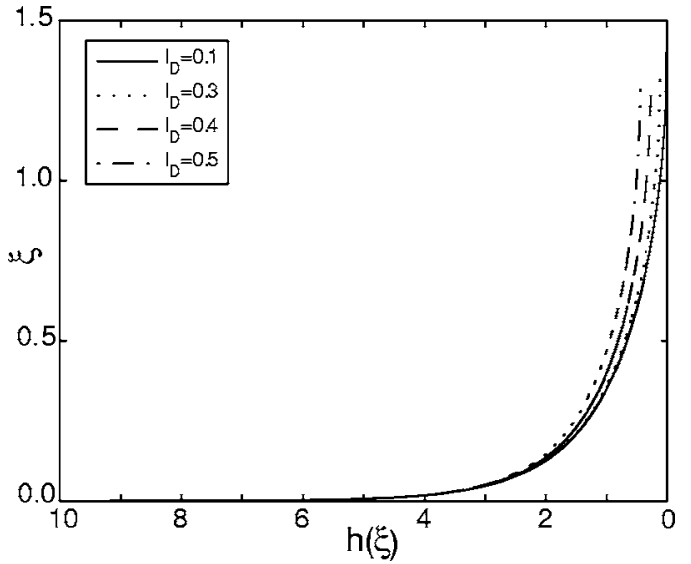

FIG. 2. Meniscus shape: attractive interaction; $\lambda_{0}=-1.0$.

As discussed previously, the lack of this invariance substantially changes the procedure of constructing the solution. The film thickness is dictated not by matching the solution in the dynamic meniscus region to the curvature in the static one as done classically by Landau and Levich, but by determining the distance from the static meniscus to the solid wall at the point $\xi^{*}$ where the slope vanishes. Thus, the entrained film thickness is $\mathrm{Ca}$ independent to leading order. Figure 3 gives the scaled film thickness versus $l_{D}$ for $\lambda_{0}=-1.0$. (In this and later figures, the plotting symbols give the computed points and the smooth curves are drawn to guide eye.)

Repulsive interaction, $\lambda_{0}>0$. Results for static meniscus profiles for $\lambda_{0}=1.0$ for various $l_{D}$ are shown in Fig. 4 . The case of repulsive potentials yield two different behaviors. There is a range of $l_{D} \in[0,0.09]$ where the point of tangency lies within the fluid, in which case the film thickness is determined as for attractive potentials, and leads to velocityindependent film thicknesses to leading order. The results show that the film thickness decreases with increasing $l_{D}$, i.e., this case exhibits velocity-independent film thinning.

Perhaps more interestingly, Fig. 4 also shows a range of parameters for which the static meniscus intersects the plate at a finite apparent contact angle. (Recall that the static meniscus is not invariant to a shift in $h$, so the tangency point cannot be made to lie within the fluid.) It is notable that this

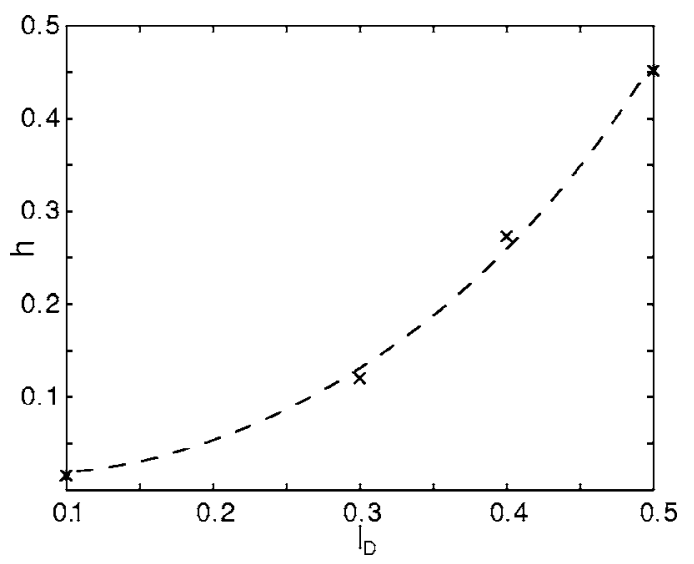

FIG. 3. Attractive potential: nondimensional film thickness $h$ is determined by static meniscus solution and is $\mathrm{Ca}$ independent.

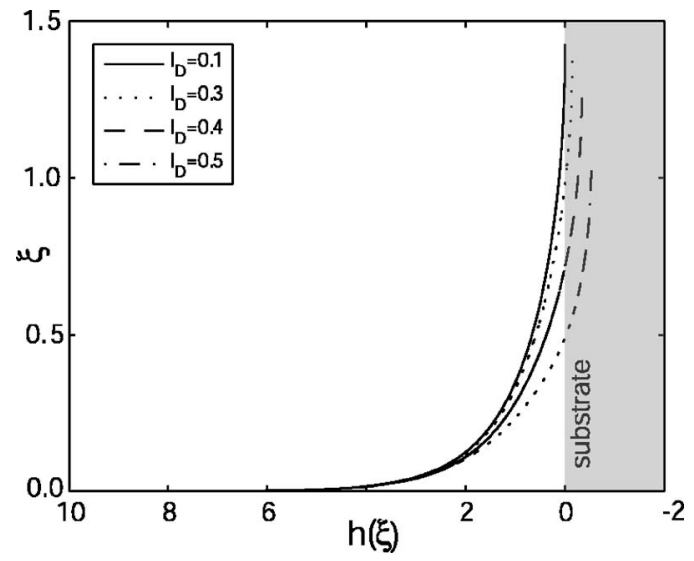

FIG. 4. Meniscus shape: repulsive interaction; $\lambda_{0}=1.0$.

is visually similar to a finite contact angle, but the physics and implication for film entrainment are different. The partial wetting case (a) is due to the force at the solid-liquid interface, as accounted for by the Young equation (3), whereas in our case (b) the finite contact angle is due to the bulk force. Thus when the film is entrained in case (a), the film effectively makes the wetting perfect, and thus the Landau-Levich procedure is valid. In our case (b) the meniscus retains its shape and does not correspond to the "perfect wetting" case. In this regime the film thickness is determined through matching of the curvature in a fashion similar to the classical case, which we refer to as "modified Landau-Levich matching," and which leads to a speed-dependent coating thickness. A map of regions in the $\left(\lambda_{0}-l_{D}\right)$ plane where the film thickness is determined by either mechanism is given in Fig. 5. As can be seen, strong potentials acting over a short distance have the same effect as weaker ones with longer ranges.

Figure 6 gives the dependence of the curvature of the static meniscus at the point of intersection with the wall (see Fig. 4) as a function of $l_{D}$. In this case the film thickness is Ca dependent and defined by the formula analogous to (17), i.e., the numerical coefficient in (17) is defined as $h^{*}$

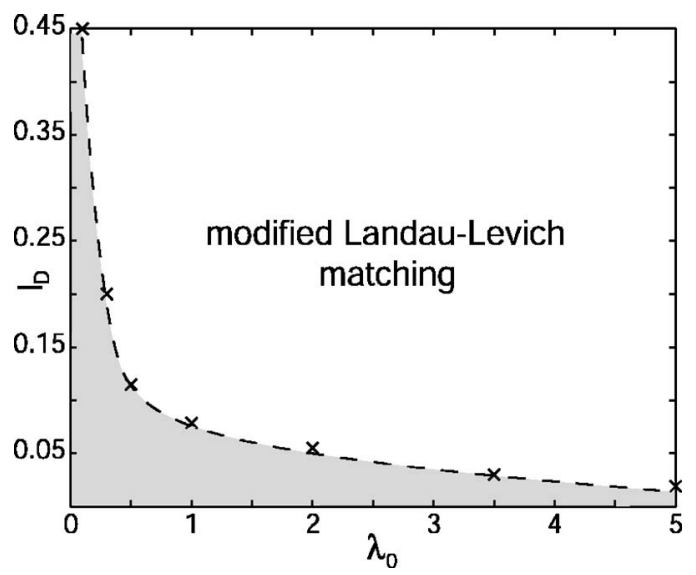

FIG. 5. Map of regimes for repulsive potentials. In the shaded region the film thickness is defined by the static meniscus solution, whereas in the modified Landau-Levich matching region the solution is constructed by matching the curvatures of the dynamic and static menisci. 


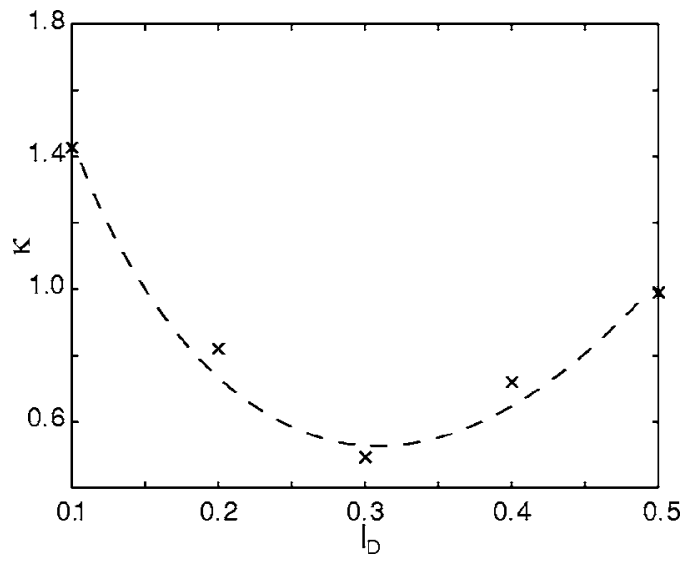

FIG. 6. Behavior of curvature at the point where meniscus approaches the substrate in the case of repulsive interaction, $\lambda_{0}=1.0$.

$=1.34 / \kappa$, where $\kappa$ is a curvature at the point where the outer static meniscus appears to intersect the substrate. Figure 7 gives the dependence of the coefficient $h^{*}$ vs $l_{D}$ for fixed $\lambda_{0}$ and indicates film thickening relative to the classical case for $l_{D}$ increasing up to the critical value around 0.3 and subsequent thinning after it.

\section{SUMMARY}

We considered the qualitative consequences of the model for dip coating in the presence of an extra body force; specifying a particular interaction potential $\phi(y, h)$, while allowing a quantitative study of a particular physical situation, will not change the general conclusions drawn here. As shown, the solution structure is very different from the approach of Teletzke et al., ${ }^{13}$ who considered the interaction term only in the dynamic meniscus region to obtain an augmented Landau-Levich equation. Rather, in the low capillary number and strong potential regime the contribution of the interaction is in the leading order in the static meniscus, whereas the dynamic meniscus stays unaffected due to this extra body force.

The primary effect of a body force normal to the substrate surface is through modification of the static meniscus

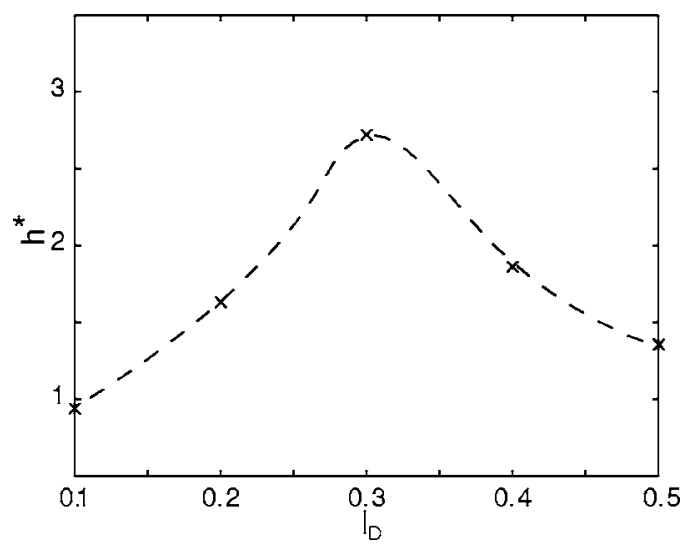

FIG. 7. Repulsive potential, $\lambda_{0}=1$ : the nondimensional film thickness is determined by modified Landau-Levich matching, and is given by $h$ $=h^{*} \mathrm{Ca}^{2 / 3}$. shape and prohibition of invariance of the solution with respect to the transformation $h \rightarrow h+$ const, a property which is important for implementing the classical matching procedure. This results in the film thickness being dictated not by matching curvature but by determining the distance from the static meniscus to the solid wall at the point of vanishing slope. This phenomenon is inherent in both attractive and repulsive interactions and leads to a coating thickness which is independent of speed to leading order. Depending on the strength of the interaction in the case of repulsive interaction, there is also a transition to the regime when the static meniscus meets the substrate at finite angle and the matching procedure involves the dynamic meniscus through a matching procedure similar to the Landau-Levich one. In this case the film thickness becomes a function of capillary number with a modified leading order coefficient. Repulsive interaction leads to nonmonotonic behavior in film thickness: for limited range of interaction $l_{D}$ one observes a film thickening relative to the classical case and film thinning for longer ranges $l_{D}$.

The intent of this work is to analyze generic nontrivial deviations from the Landau-Levich law in the presence of an extra body force. The verification of this theory along with more quantitative analysis should be motivated by actual experiments and careful definition of an interaction potential. Analytical studies of existence and uniqueness of solutions for (14) would also provide more insights. There are also some interesting features that are beyond the scope of the current work. In particular, the sudden change in the type of matching when potentials are included, $\lambda_{0} \neq 0$, suggests that as $\lambda_{0} \rightarrow 0$ one encounters a distinguished limit when both static and dynamic menisci effects become equally important. In the case $\lambda_{0}=O(1)$ the static meniscus effects, as demonstrated here, dominate the dynamic meniscus ones for attractive and a certain range of repulsive potentials. In the case of repulsive potentials there is a region in the neighborhood of the dividing curve in Fig. 5 which requires special treatment to provide a smooth transition from a film whose thickness is a function of capillary number to one independent of withdrawal speed. Finally, we have not accounted for the mechanisms by which the static shapes can be reconciled with the prediction of a finite thickness film that extends upward to infinity. The manner is which the moving substrate sustains this film may also be subtle. All of these issues are worth further studying.

\section{ACKNOWLEDGMENT}

This work was supported by the Office of Basic Energy Sciences, U. S. Dept. of Energy.

${ }^{1}$ L. Landau and B. Levich, "Dragging of a liquid by a moving plate," Acta Physicochim. URSS 17, 42 (1942).

${ }^{2}$ J. M. Davis, "Asymptotic analysis of liquid films dip-coated onto chemically micropatterned surfaces," Phys. Fluids 17, 038101 (2005).

${ }^{3}$ S. D. R. Wilson, "Coating flow on to rods and wires," AIChE J. 34, 1732 (1988).

${ }^{4}$ S. D. R. Wilson, "The drag-out problem in film coating theory," J. Eng. Math. 16, 209 (1982)

${ }^{5}$ A. D. Read and J. A. Kitchener, "The thickness of wetting films," in "Wetting," J. Soc. Chem. Ind., London 25, 300 (1967). 
${ }^{6}$ A. D. Read and J. A. Kitchener, "Wetting films on silica," J. Colloid Interface Sci. 30, 391 (1969).

${ }^{7}$ T. M. Squires and M. P. Brenner, "Like-charge attraction and hydrodynamic interaction," Phys. Rev. Lett. 85, 4976 (2000).

${ }^{8}$ S. H. Behrens and D. G. Grier, " The charge of glass and silica surfaces," J. Chem. Phys. 115, 6716 (2001).

${ }^{9}$ R. K. Iler, The Chemistry of Silica (Wiley, New York 1979).

${ }^{10}$ J. F. Padday, "Cohesive properties of thin films of liquids adhearing to a solid surface," in Thin Liquid Films and Boundary Layers (Special Discussions of the Faraday Society) (Academic, New York, 1970), pp. 64-88.

${ }^{11}$ V. S. Mitlin, " Dewetting of solid surface: analogy with spinodal decomposition,” J. Colloid Interface Sci. 156, 491 (1993).

${ }^{12}$ J. A. Tallmadge and R. Stella, "Some properties of the apparent water paradox in entrainment," AIChE J. 14, 838 (1968).

${ }^{13}$ G. F. Teletzke, H. T. Davis, and L. E. Scriven, "Wetting hydrodynamics," Rev. Phys. Appl. 23, 989 (1988).

${ }^{14}$ F. P. Bretherton, “ The motion of long bubbles in tubes," J. Fluid Mech. 10, 166 (1961).

${ }^{15}$ The choice of the potential $\phi$ is not trivial. In the works of Ruckenstein and Jain (Ref. 18). Williams and Davis (Ref. 19), Teletzke and co-workers (Ref. 13), the form $\phi=A / h^{3}$ is used, which corresponds to an interfacial potential and thus cannot be simply inserted into the bulk equations. At the same time, redesignation $h \rightarrow y$ is not valid either since in the layer the form of the potential is different. By their nature, London-van der Waals forces are bulk forces and, in general, the functionality of $\phi$ should involve both dependencies on $y$ and $h(x)$, since the potential depends not only on the film thickness, but on the position within the film. This is crucial in getting to the augmented Landau-Levich equation since the derivation procedure involves integration over the thin dimension.

${ }^{16}$ C.-W. Park and G. M. Homsy, " Two-phase displacement in Hele-Shaw cells: theory,” J. Fluid Mech. 139, 291 (1984).

${ }^{17}$ This impossibility was also verified by an alternative method of integrating (14) as a multiparameter shooting in the unstable direction (bath interface) from the end-point located at the substrate, which showed that asymptotic behavior (20) is not achievable in general. We also investigated other approaches to solve (14) such as an introduction of an arclength formulation, $\mathrm{d} s=\sqrt{1+h_{\xi}^{2}} \mathrm{~d} \xi$, which allows even further reduction, $h_{s}=f(h)$, but all of them lead to the appearance of the term $\sqrt{1-h_{s}^{2}}$, which numerically moves the solution into the complex plane since the shooting should reach the boundary, $\left|h_{s}\right|=1$, with singular (complex) solution defined by $\left(\left|h_{s}\right|=|f|>1\right)$.

${ }^{18}$ E. Ruckenstein and R. K. Jain, "Spontaneous rupture of thin liquid films," J. Chem. Soc., Faraday Trans. 1 70, 132 (1974).

${ }^{19}$ M. B. Williams and S. H. Davis, "Nonlinear theory of film rupture," J. Colloid Interface Sci. 90, 220 (1982). 